\title{
The Half-Filled One-Dimensional Extended Hubbard Model: Phase diagram and Thermodynamics
}

\author{
S. Glocke, ${ }^{1}$ A. Klümper, ${ }^{1}$ and J. Sirker ${ }^{2}$ \\ ${ }^{1}$ Bergische Universität Wuppertal, Fachbereich Physik, 42097 Wuppertal, Germany \\ ${ }^{2}$ Max-Planck-Institute for Solid State Research, Heisenbergstr. 1, 70569 Stuttgart, Germany
}

(Dated: August 23, 2018)

\begin{abstract}
We study the thermodynamics of the one-dimensional extended Hubbard model at half-filling using a density-matrix renormalization group method applied to transfer matrices. We show that the various phase transitions in this system can be detected by measuring usual thermodynamic quantities like the isothermal compressibility and the uniform magnetic susceptibility. For the isothermal compressibility we show that universal crossing points exist which allow to accurately determine the line where the charge gap vanishes. By studying in addition several correlation functions, we confirm the existence of a phase with long-range dimer order (bond order) which has been a matter of debate for several years. According to our calculations this phase is located in a narrow region between the spin-density and charge-density wave phases up to a tricritical point which we estimate to be at $U_{t}=6.7 \pm 0.2, V_{t}=3.5 \pm 0.1$. Our results for the phase diagram are in good agreement with the most recent zero-temperature density-matrix renormalization group study, however, they disagree in some important aspects from the most recent Quantum-MonteCarlo study.

PACS numbers: 71.10.Fd, 71.10.Pm, 71.10.Hf, 05.70.-a
\end{abstract}

\section{INTRODUCTION}

Understanding the effects of competing interactions and the associated quantum phase transitions in models of strongly correlated electron systems is still one of the main issues of modern condensed matter physics. In one dimension correlation effects are particularly strong and a number of analytical and numerical tools have been developed for this case. This has led to intense work on such systems in the last decades. One of the seminal models in this context is the extended Hubbard model

$H=-t \sum_{j, \sigma}\left(c_{j, \sigma}^{\dagger} c_{j+1, \sigma}+\right.$ h.c. $)+U \sum_{j}\left(n_{j, \uparrow}-\frac{1}{2}\right)\left(n_{j, \downarrow}-\frac{1}{2}\right)+V \sum_{j}\left(n_{j}-1\right)\left(n_{j+1}-1\right)-\frac{h}{2} \sum_{j}\left(n_{j, \uparrow}-n_{j, \downarrow}\right)-\mu \sum_{j} n_{j}$.

Here $c_{j, \sigma}^{\dagger}\left(c_{j, \sigma}\right)$ is a creation (annihilation) operator for an electron with spin $\sigma=\uparrow, \downarrow$ at site $j, n_{j, \sigma}=c_{j, \sigma}^{\dagger} c_{j, \sigma}$, and $n_{j}=n_{j, \uparrow}+n_{j, \downarrow} . t$ is the amplitude of a nearest neighbor hopping, $U \geq 0$ an on-site and $V \geq 0$ a nearest-neighbor Coulomb repulsion. A possible additional magnetic field is denoted by $h$ and the chemical potential by $\mu$. Here we will concentrate on the half-filled case $\mu=0$. In the following, we will only keep the hopping amplitude $t$ where it is necessary for the sake of clarity and set $t=1$ otherwise.

In the strong coupling limit, $U, V \gg t$, it is easy to see by simple energetical considerations that two different ground states exist: For $U<2 V$ the system is an insulator with long-range charge density wave (CDW) order whereas for $U>2 \mathrm{~V}$ a state with quasi-long-range spin density wave (SDW) order forms. The transition between these two phases in the strong coupling limit is first order. ${ }^{1-3}$ In the weak coupling limit, $U, V \ll t$, the model can be studied using bosonization and g-ology. ${ }^{4,5}$ In this framework one finds again a phase transition between the SDW and CDW phase at $U=2 V$. In the spin sector this transition is driven by an operator which turns from marginally irrelevant in the SDW phase to marginally relevant in the CDW phase. The spin gap therefore opens up exponentially slowly and the transition in the spin sector is of Kosterlitz-Thouless (KT) type. In the charge sector, on the other hand, there is a relevant operator in both phases leading to a charge gap. The amplitude of this operator vanishes only at the transition line $U=2 \mathrm{~V}$ so that the charge gap disappears. ${ }^{4}$ The transition in the charge sector is therefore second order. Already from the strong and weak coupling approaches it is clear that a point $\left(U_{t}, V_{t}\right)$ in the intermediate coupling regime must exist where the order of the phase transition changes.

In the last few years the extended Hubbard model has attracted renewed attention because it has been suggested that the phase diagram obtained by the weak coupling g-ology approach and strong-coupling perturbation 
theory might not be complete. Nakamura pointed out first that there is no symmetry requiring the lines in $U, V$-parameter space, where the marginal operator in the spin sector changes sign and where the relevant operator in the charge sector vanishes, to coincide. ${ }^{4,6}$ The coupling constants for these two operators do coincide in the standard g-ology approach where they are calculated to first order in the interaction parameters. However, they might differ once higher order corrections are taken into account. This opens up the possibility for an intermediate phase. By extracting the scaling dimensions related to the critical exponents of certain correlation functions from finite size energy spectra, Nakamura indeed found a phase with long-range dimer order in a small region between the SDW and CDW phases. This phase is often called a bond-order wave (BOW) state. The existence of such a phase around $U=2 \mathrm{~V}$ in the weak coupling regime was supported by Quantum-Monte-Carlo (QMC) calculations ${ }^{7,8}$ as well as by a g-ology approach where the coupling constants have been calculated beyond leading order. ${ }^{9}$ However, in a first DMRG calculation ${ }^{10}$ such a phase was only found above the tricritical point $\left(U_{t}, V_{t}\right)$ and only directly at the first order transition line. A later DMRG calculation, ${ }^{11}$ on the other hand, qualitatively confirms again the phase diagram as proposed by Nakamura. Further evidence for the existence of a BOW phase in the weak coupling regime was also provided by a functional renormalization group analysis. ${ }^{12}$

Although the most recent DMRG ${ }^{11}$ and the most recent QMC study ${ }^{8}$ agree that a BOW phase of finite extent does exist, they disagree about the shape of this phase. Whereas in the phase diagram of Ref. 11 the BOW phase ends at the tricritical point, it extends beyond this point to larger values of $U, V$ in the phase diagram of Ref. 8. The question whether the tricritical point also marks the end of the BOW phase or is located on the BOW-CDW boundary therefore remains an open issue.

In this work we will investigate the half-filled onedimensional extended Hubbard model using a densitymatrix renormalization algorithm applied to transfer matrices (TMRG). This numerical method allows it to calculate thermodynamic properties of the model in the thermodynamic limit. We will provide further evidence for the correctness of the phase diagram as first proposed by Nakamura and give an estimate for the tricritical point $\left(U_{t}, V_{t}\right)$. In particular, we will argue based on our numerical results that the BOW phase ends at the tricritical point and does not extend to larger values of $U, V$ in contrast to the findings in Ref. 8. In the process, we will develop and discuss criteria to identify the different phases and transition lines by considering usual thermodynamic quantities like the uniform magnetic susceptibility, the isothermal compressibility (charge susceptibility), and the specific heat.

Our paper is organized as follows: In Sec. II we briefly introduce the TMRG algorithm and compare results for the Hubbard model $(V=0)$ with exact results obtained by the Bethe ansatz. In Sec. III we then present results for a variety of thermodynamic quantities which allow us to determine the phase diagram of the extended Hubbard model at half-filling. The last section is devoted to our conclusions.

\section{THE TMRG ALGORITHM AND THE HUBBARD MODEL}

The density-matrix renormalization group applied to transfer matrices (TMRG) has been explained in detail in $[13,14,15]$. Here we only want to briefly discuss the most important aspects. The TMRG algorithm is based on a mapping of a one-dimensional quantum system to a twodimensional classical one by means of a Trotter-Suzuki decomposition. In the classical model one direction is spatial whereas the other corresponds to the inverse temperature. For the classical system a so called quantum transfer matrix (QTM) is defined which evolves along the spatial direction. At any non-zero temperature the QTM has the crucial property that its largest eigenvalue $\Lambda_{0}$ is separated from the other eigenvalues by a finite gap. The partition function of the system in the thermodynamic limit is therefore determined by $\Lambda_{0}$ only, allowing it to perform this limit exactly. The Trotter-Suzuki decomposition is discrete so that the transfer matrix has a finite number of sites or local Boltzmann weights $M$. The temperature is given by $T \sim(\epsilon M)^{-1}$ where $\epsilon$ is the discretization parameter used in the Trotter-Suzuki decomposition. The algorithm starts at some high-temperature value where $M$ is so small that the QTM can be diagonalized exactly. Using a standard infinite-size DMRG algorithm, sites are then added to the QTM leading to a successive lowering of the temperature. A source for a systematic error in these calculations is the finite discretization parameter $\epsilon$. However, this only leads to errors of order $\epsilon^{2}$ in all thermodynamic quantities considered in the following. We will choose $\epsilon=0.025$ or 0.05 so that this systematic error will only be of order $10^{-3}-10^{-4}$. Another error is introduced by the truncation of the Hilbert space. This error will grow with decreasing temperature and will finally make the calculations unreliable. Down to which temperature the DMRG algorithm works will depend on the maximum number of states $N$ kept in the truncated Hilbert space basis. The truncation error is difficult to estimate. We therefore start by comparing our TMRG results for the Hubbard model $(V=0)$ with exact results obtained by Bethe ansatz. ${ }^{16}$ Within the TMRG algorithm nothing changes fundamentally when we introduce the nearest-neighbor Coulomb repulsion $V$ so that we expect a similar accuracy in this case.

As an example, we consider the case $U=8$. Results with a similar accuracy are also obtained for other $U$. Using the TMRG method, the free energy per site is given by

$$
f=-T \ln \Lambda_{0}
$$

The specific heat is then obtained by $C=-T \partial^{2} f / \partial T^{2}$ 


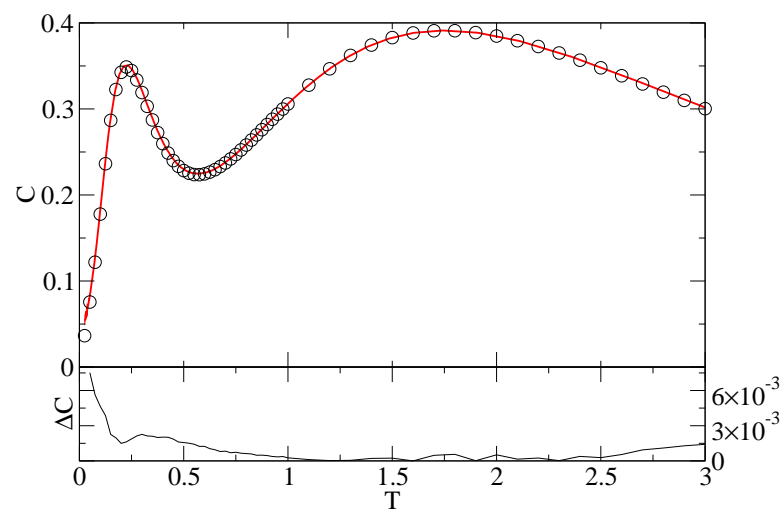

FIG. 1: (Color online) TMRG data for the specific heat $C$ of the Hubbard model at $U=8$ with $N=200$ states kept (red solid line) compared to Bethe ansatz data (circles) as a function of temperature $T$. The lower graph shows the error $\Delta C$ of the TMRG calculation.

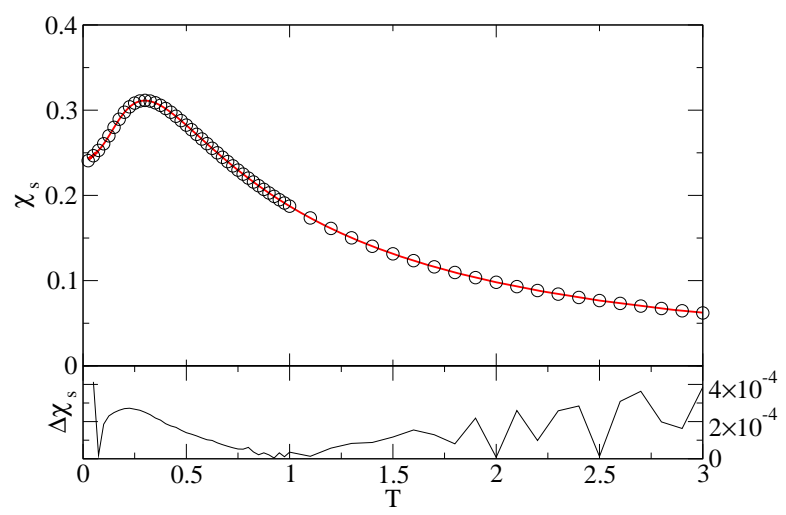

FIG. 2: (Color online) Same as Fig. 1 for the magnetic susceptibility $\chi_{s}$.

and is shown in Fig. 1. It is also easy to calculate the expectation values of local operators with the TMRG algorithm. To obtain the magnetic susceptibility, $\chi_{s}$, the expectation value $m \equiv\left\langle S^{z}\right\rangle=\left\langle n_{\uparrow}-n_{\downarrow}\right\rangle / 2$ is calculated in the presence of a small magnetic field $\delta h \sim 10^{-2}$. The susceptibility is then given by $\chi_{s}=m / \delta h$ and shown in comparison to the exact result in Fig. 2. Similarly, the isothermal compressibility (charge susceptibility), $\chi_{c}$, is obtained by applying a small chemical potential $\delta \mu$ and is shown in Fig. 3. For the spin and charge susceptibilities $\chi_{s}, \chi_{c}$ the error does not exceed $5 \times 10^{-4}$ down to temperatures $T \approx 0.05$. For the specific heat $C$, the errors are an order of magnitude larger because a second numerical derivative has to be calculated.

\section{THE PHASE DIAGRAM OF THE EXTENDED HUBBARD MODEL AT HALF-FILLING}

To investigate the phase diagram we will consider a number of different thermodynamic quantities like mag-

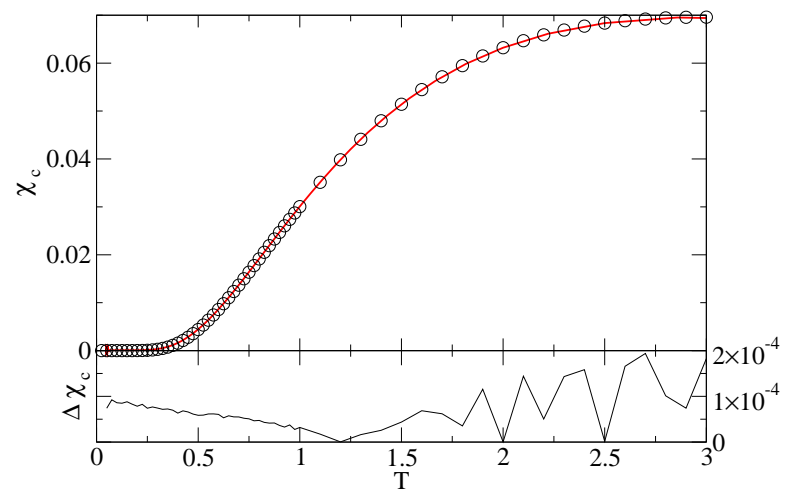

FIG. 3: (Color online) Same as Fig. 1 for the charge susceptibility $\chi_{c}$.

netic susceptibilities, compressibilities, specific heats, and expectation values of local operators. Furthermore, we will study the behavior of correlation lengths which can be obtained within the TMRG method by calculating next-leading eigenvalues of the QTM. ${ }^{13,15}$ Depending on the required accuracy and the temperature regime we want to access the basis for the truncated Hilbert space will consist of $N=200-400$ states.

We start with the strong coupling limit where the two existing phases and the first order phase transition between these phases are well understood. We then derive an estimate for the tricritical point where the first order line ends. Next we discuss the considerably more complicated weak coupling regime and present the phase diagram as obtained by TMRG. Finally, we will address the controversial question whether or not the BOW phase ends at the tricritical point. Throughout, we will discuss in how far one can identify the different phases and phase transitions by studying only easily measurable thermodynamic quantities like the specific heat, magnetic susceptibility and compressibility.

\section{A. Strong coupling}

In the strong coupling limit, $U, V \gg t$, the ground state energy can be systematically expanded in terms of the hopping parameter $t$. In lowest order, the hopping can be completely neglected. Then, depending on the ratio $U / V$, two different ground states are possible. These states are depicted in Fig. 4. The energy of the CDW state is then given by $E_{C D W}^{0}=L U / 4-L V$ with $L$ being the number of lattice sites. The SDW state has energy $E_{S D W}^{0}=-L U / 4$. The two energies as a function of $U, V$ cross at $U=2 V$ resulting in a first order phase transition. As usual, in second order in $t$, virtual hopping processes lead to an effective antiferromagnetic coupling of Heisenberg type for the spins in the SDW state with coupling constant $J=2 t^{2} /(U-V) .^{1,3}$ This state therefore has a charge gap but no spin gap and algebraically decaying spin correlation functions. The CDW state, on the 


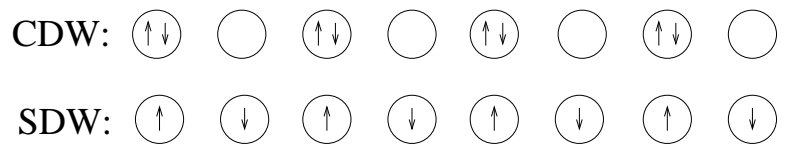

FIG. 4: The two ground states in the strong coupling limit $U, V \gg t$. The state in the first line is a CDW state where every second site is doubly occupied, whereas the state in the second line is a state with every site singly occupied. Virtual hopping processes induce a quasi long-range SDW order for this state.

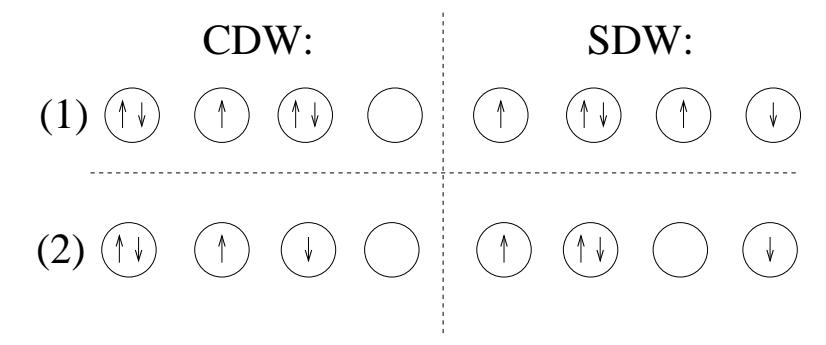

FIG. 5: Left column: (1) CDW state with one particle added, (2) CDW state with one double occupancy broken. Right column: (1) SDW state with one particle added, (2) SDW state with one double occupancy.

other hand, has a charge and a spin gap. Excitations for the CDW and SDW state, ignoring hopping processes, are shown in Fig. 5. In lowest order perturbation theory, the energies of the excited states depicted in Fig. 5 are given by $E_{C D W}^{1}=E_{C D W}^{0}-U / 2+2 V, E_{C D W}^{2}=$ $E_{C D W}^{0}-U+3 V$ for the excited CDW states, and $E_{S D W}^{1}=E_{S D W}^{0}+U / 2, E_{S D W}^{2}=E_{S D W}^{0}+U-V$ for the excited SDW states. Excitation (1) in the CDW state is a charge excitation, whereas the breaking of a double occupancy - excitation (2) - is a spin excitation. If we separate the two single spins in this excited state we obtain an excitation energy $E_{C D W}^{0}+2(-U / 2+2 V)$, i.e., each single spin contributes $-U / 2+2 V$. In thermodynamic data the activated behavior will be characterized by the energy of a single excitation irrespective of whether these excitations appear in pairs or not. In the strong coupling limit, it follows that at the transition line charge and spin gap as obtained from thermodynamic data are expected to be equal $\Delta_{s}=\Delta_{c}=U / 2$ and that both gaps increase linearly $\sim 2 V$ away from the transition line. In the SDW phase, excitation (1) is also a charge excitation and has a lower energy than excitation (2). The charge gap in the SDW phase is therefore given by $\Delta_{c}=U / 2$ and is independent of $V$.

In Fig. 6, TMRG results for the spin susceptibility $\chi_{s}$ and the spin gap $\Delta_{s}$ at $U=12$ are shown. If a gap $\Delta$ exists, the dispersion of the elementary excitations is given by $\epsilon(k) \sim \Delta+k^{2} /(2 m)$ with some effective mass $m$. It is then easy to see that the corresponding susceptibility will show activated behavior

$$
\chi(T) \sim \frac{\exp (-\Delta / T)}{\sqrt{T}}
$$

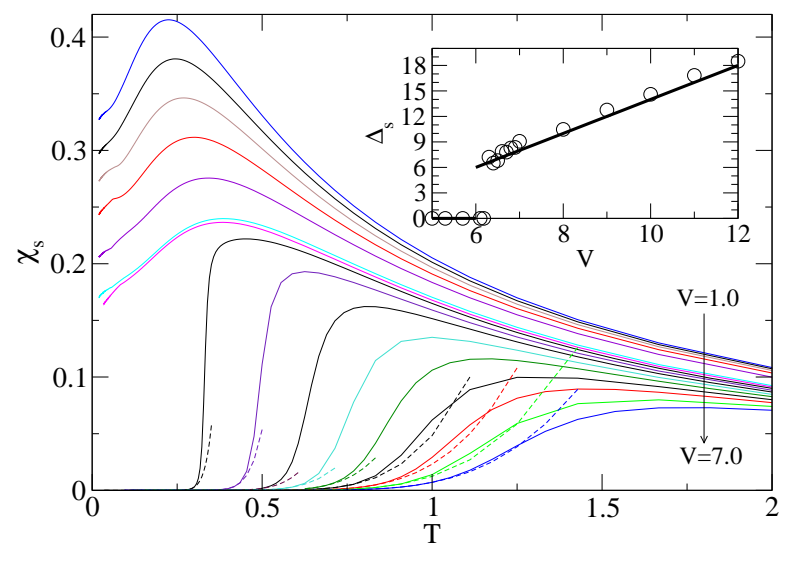

FIG. 6: (Color online) Magnetic susceptibilities (solid lines) for $U=12$ and $V=1.0,2.0, \cdots, 6.0,6.1,6.2, \cdots, 7.0$ as a function of temperature $T$. The dashed lines are fits according to Eq. (3). The inset shows the spin gap $\Delta_{s}$ extracted from those fits (circles) as a function of $V$. The solid lines in the inset denote the theoretical result in the strong coupling limit.

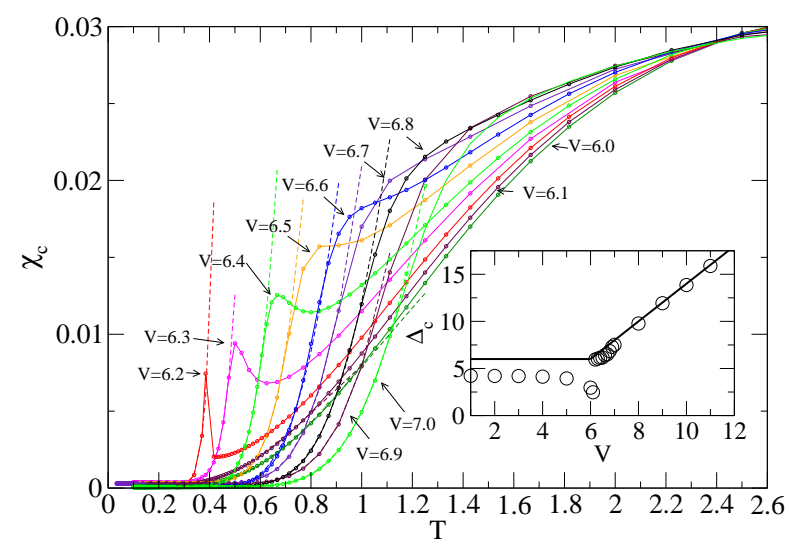

FIG. 7: (Color online) Charge susceptibilities (circles) for $U=$ 12 and $V=6.0,6.1, \ldots, 7.0$ as a function of temperature $T$. The lines are a guide to the eye. The dashed lines are fits according to Eq. (3). The inset shows the charge gap $\Delta_{c}$ extracted from those fits (circles) as a function of $V$. The solid lines in the inset denote the theoretical result in the strong coupling limit.

at temperatures $T \ll \Delta$. Using this function to fit the numerical data, we are able to extract the spin gap $\Delta_{s}$. As shown in the inset of Fig. 6 the behavior of $\Delta_{s}$ as a function of $V$ at $U=12$ is already reasonably well described by the strong coupling limit, i.e., there is no spin gap up to $V \approx U / 2$, then $\Delta_{s}$ jumps to approximately $U / 2$ and then increases linearly with slope 2 .

Similarly, we show TMRG results for the charge susceptibility $\chi_{c}$ and the charge gap $\Delta_{c}$ at $U=12$ in Fig. 7 . The results obtained for the charge gap $\Delta_{c}$ are also already close to the strong coupling limit, although the gap is a bit smaller than $U / 2$ in the SDW phase and it shows some $V$ dependence when the transition point is approached. 


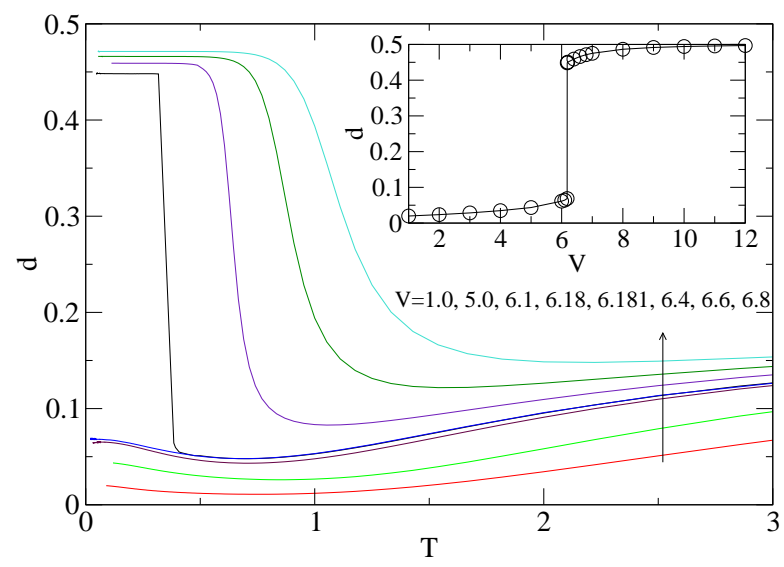

FIG. 8: (Color online) Double occupancy as a function of temperature for $U=12$ and different $V$. Inset: Extrapolated values of the double occupancy at zero temperature as a function of $V$.

Another quantity which allows us to detect the phase transition and to determine its order is the double occupancy

$$
d=\left\langle n_{j, \uparrow} n_{j, \downarrow}\right\rangle .
$$

In the strong coupling limit at zero temperature $d=0$ in the SDW state and $d=1 / 2$ in the CDW state. In Fig. 8 we show $d$ for $U=12$ and various $V$. In the extrapolated data for zero temperature some corrections to the strong coupling limit are visible. $d$ is already nonzero in the SDW phase and increases slightly with $V$. However, a jump in $d$ at $V \approx 6.18$ is obvious. In the CDW phase $d$ continues to increase with $V$ and approaches $1 / 2$ in the large $V$ limit.

The specific heat shown in Fig. 9 has two maxima for $U=12$ and $V=0$. The lower and higher temperature maximum are due to spin and (gapped) charge excitations, respectively. ${ }^{17}$ At low temperatures only the gapless spin excitations do therefore contribute and conformal field theory predicts

$$
C=\frac{\pi}{3 v_{s}} T
$$

With increasing $V$ the spin velocity $v_{s}$ increases leading to a decreasing slope and to a shift of the lower temperature maximum to higher temperatures. At the same time the charge gap decreases leading to a shift of the higher temperature maximum to lower temperatures. The behavior changes drastically above the phase transition $V>V_{c} \approx 6.18$, because in the CDW phase the spin excitations are now also gapped and the specific heat shows activated behavior $C \sim e^{-\Delta / T}$ with $\Delta=\min \left(\Delta_{s}, \Delta_{c}\right)$. The emergence of a sharp peak for $V \gtrsim V_{c}$ can be understood as follows: Because $\int_{0}^{\infty} C(T) d T=-e_{0}$ with $e_{o}$ being the ground state energy, the area under the curve will be nearly unchanged when going from a value just below the phase transition, say $V=6.1$, to a value just

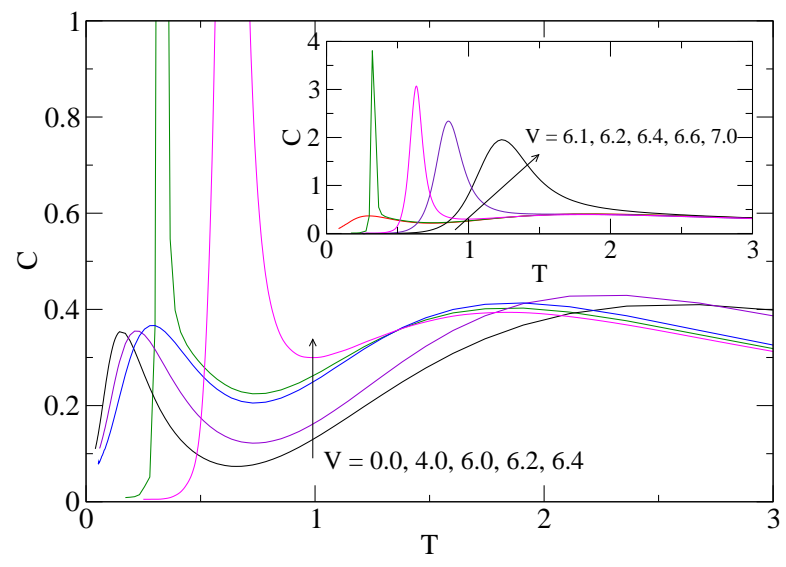

FIG. 9: (Color online) Specific heat as a function of temperature for $U=12$ and different $V$. Inset: A sharp peak forms just above phase transition.

above the transition, say $V=6.2$. In addition, also the high temperature behavior will be almost unaffected by this small change in $V$. Hence the weight suppressed by the gap at low temperatures will show up in a sharp peak just above the gap. This is shown in the inset of Fig. 9 and constitutes one possibility to detect the first order transition easily from thermodynamic data.

\section{B. The tricritical point}

From the discussion in the introduction it is clear that the first order transition line must end at some point $\left(U_{t}, V_{t}\right)$ because the phase transitions at weaker couplings are expected to be continuous. We found that a good criterion to determine this endpoint with the TMRG method is to study the double occupancy $d(4)$. As shown for the case $U=12$ in Fig. 8, $d$ as a function of $T$ shows a dramatically different behavior depending on weather we choose a $V$ such that we are in the SDW phase or a $V$ such that we are in the CDW phase. $d$ at a fixed $U$ extrapolated to zero temperature therefore shows a jump $\Delta_{d}$ as a function of $V$ if the phase transition is first order. Reducing the on-site repulsion $U$ we expect this jump to become smaller and smaller until it disappears at $U_{t}$. For $U=7.0$ we can still detect a finite jump $\sim 0.17$ at $V \approx 3.65$ (see Fig. 10(c)) whereas $d$ as a function of $V$ seems to be continuous for $U=6$ (see Fig. 10(b)). To determine the point $\left(U_{t}, V_{t}\right)$ more accurately we have plotted the jump $\Delta_{d}$ as a function of $U$ in Fig. 10(d). We can fit these data very well by a power law which leads us to the estimate $U_{t}=6.7 \pm 0.2$. Because the value for $U=7$ is least reliable, we also did fits where this point was excluded. Similarly, we tried fits where the data points for large $U$ were excluded. The results of the various fits lead to the error estimate above. For each possible value of $U_{t}$ we can find $V_{t}$ with high accuracy. For the values of $U_{t}$ estimated above, we have $V_{t}=3.5 \pm 0.1$. Here the uncertainty in $V_{t}$ is not an error 


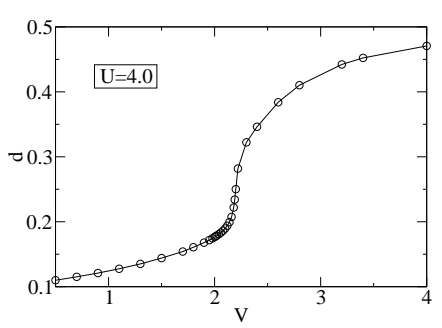

(a) $d$ for $U=4.0$

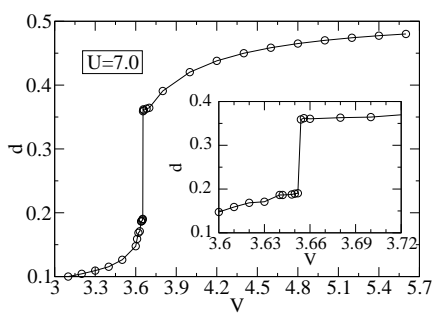

(c) $d$ for $U=7.0$

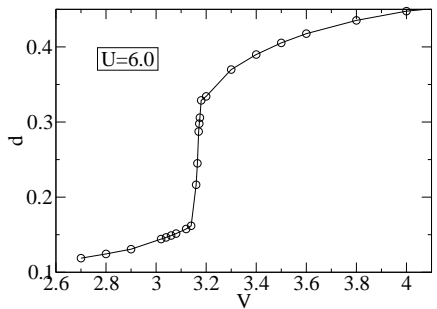

(b) $d$ for $U=6.0$

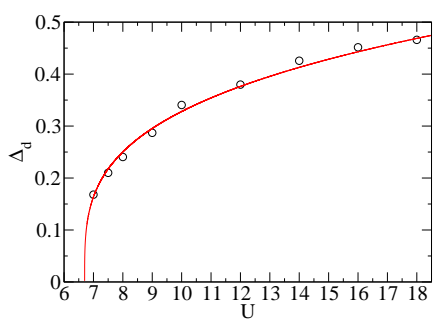

(d) $\Delta_{d}(U)$
FIG. 10: (Color online) Extrapolated values of the double occupancy $d$ at zero temperature as a function of $V$ for different $U$. The inset of (c) shows a zoom of the region where $d$ jumps. (d) Extrapolated TMRG data (circles) for the jump $\Delta_{d}$ in the double occupancy at the phase transition at zero temperature as a function of $U$. The line is a fit $\Delta_{d}=0.232(U-6.7)^{0.29}$.

estimate but rather means that $V_{t} \approx 3.4$ for $U_{t}=6.5$ and $V_{t} \approx 3.6$ for $U_{t}=6.9$.

\section{Weak coupling}

The phase diagram in the weak coupling limit is more complicated than in the strong coupling limit. Instead of a first order, we expect different continuous phase transitions here. Theoretically, the weak-coupling limit can be investigated by bosonization with the coupling constants of the operators in the effective Hamiltonian being determined in first order in the interaction parameters. This method is also often termed g-ology. ${ }^{5}$ As usual in one-dimension, the charge and the spin sector completely separate in the low-energy effective bosonic theory due to the linearization of the excitation spectrum. In the charge sector at half-filling, Umklapp scattering leads to a relevant interaction term in the bosonic Hamiltonian which creates a charge gap. In the spin sector, on the other hand, the leading interaction term corresponding to backward scattering is only marginal. The amplitudes of both terms in the weak-coupling limit are proportional to $U-2 V{ }^{4}$ The system therefore has always a charge gap except at $U=2 \mathrm{~V}$ where the amplitude of the Umklapp scattering term vanishes. The charge gap at fixed $U$ near the phase transition behaves as

$$
\Delta_{c} \sim\left|V-V_{c}\right|^{\alpha}
$$

with $\alpha>0$ being an interaction dependent critical exponent and $V_{c} \approx U / 2$ at weak coupling. This means that the transition in the charge sector is second order. In the spin sector at weak coupling the backward scattering term is marginally irrelevant if $U>2 \mathrm{~V}$ so that the spin excitations are gapless. For $U<2 V$ this term becomes marginally relevant and a spin gap $\Delta_{s}$ appears. However, this gap only opens up exponentially slow, i.e., for a fixed $U$ and $V \gtrsim V_{c}$ we expect

$$
\Delta_{s} \sim \sqrt{V-V_{c}} \exp \left\{- \text { const } /\left(V-V_{c}\right)\right\}
$$

with $V_{c} \approx U / 2$ at weak coupling. ${ }^{18}$ The phase transition in the spin sector is therefore of Kosterlitz-Thouless (KT) type.

As Nakamura [6] first noted, there is no symmetry which fixes the amplitude of the Umklapp and backward scattering terms to be the same. So although these amplitudes are identical to first order in the interaction parameters, one would expect in general that they start to differ once higher order corrections are taken into account. In this case an additional phase between the SDW and CDW phases would occur. As already outlined in the introduction different methods have given strong evidence that an additional phase with BOW order does indeed exist although some controversy about the extent of this additional phase remains. ${ }^{4,6-12}$

In the following, we will first develop a criterion which allows us to determine the second order line where the charge gap closes with high precision from thermodynamic data. Next, we will consider the KT-type transition where the spin gap opens. Finally, we will provide some direct evidence that the new phase has long-range BOW order at zero temperature and does not extend beyond the tricritical point.

To determine the line in the $U, V$-phase diagram where the charge gap closes, we consider the charge susceptibility $\chi_{c}$. If a charge gap $\Delta_{c}$ exists, $\chi_{c}$ at temperatures $T \ll \Delta_{c}$ is described by Eq. (3). In the low temperature regime, $\chi_{c}$ therefore will be larger the smaller the charge gap is. According to Eq. (6) we therefore expect the following behavior of $\chi_{c}\left(T_{0}, V\right)$ at fixed $U$ and fixed low temperature $T_{0}$ : If $V<V_{c}$ then $\chi_{c}\left(T_{0}, V\right)$ increases with increasing $V$ whereas $\chi_{c}\left(T_{0}, V\right)$ decreases with increasing $V$ if $V>V_{c}$.

For high temperatures $T_{0} \gg 1$, on the other hand, $\chi_{c}\left(T_{0}, V\right)$ will always decrease with increasing $V$ as can be easily seen from a high temperature expansion. Up to second order in $1 / T$ we find

$$
\chi_{c}(T \gg 1)=\frac{1}{2 T}\left[1-\frac{1}{2 T}(U / 2+V)\right] .
$$

For $V<V_{c}$ we therefore have the situation that $\partial \chi_{c} / \partial V>0$ for $T \ll 1$ and $\partial \chi_{c} / \partial V<0$ for $T \gg 1$. The compressibility curves for different $V<V_{c}$ at fixed $U$ therefore have to cross at least at one point. For $V>V_{c}$, on the other hand, we have $\partial \chi_{c} / \partial V<0$ for high as well as for low temperatures so that no crossing is expected. The different behavior of the compressibility curves for $V>V_{c}$ and $V<V_{c}$ is a very efficient criterion to determine $V_{c}$ as is shown in Figs. 11, 12 for the cases $U=2$ 

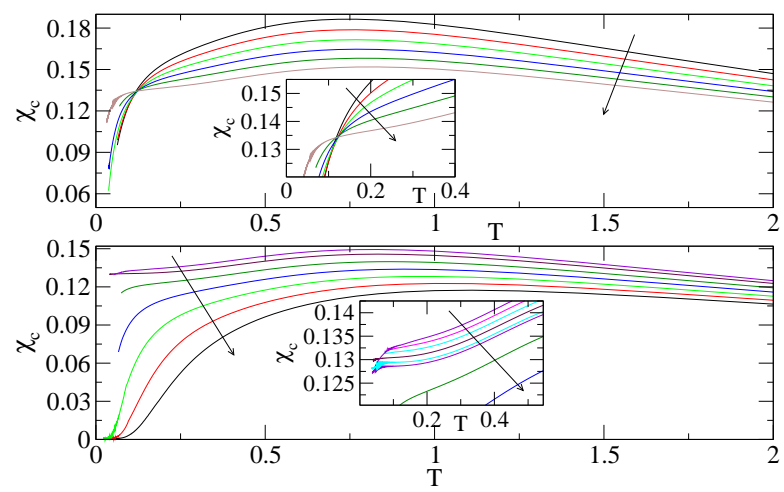

FIG. 11: (Color online) Charge susceptibility $\chi_{c}$ for $U=2$. Upper panel: $\chi_{c}$ for $V=0.5,0.6, \cdots, 1.0$ (in arrow direction). The inset shows a zoom of the region around the crossing point at $T^{*} \approx 0.12$. Lower panel: $\chi_{c}$ for $V=1.04,1.1,1.2, \cdots, 1.6$ (main, in arrow direction) and $V=1.04,1.06,1.08,1.1,1.12,1.13,1.2,1.3$ (inset, in arrow direction).
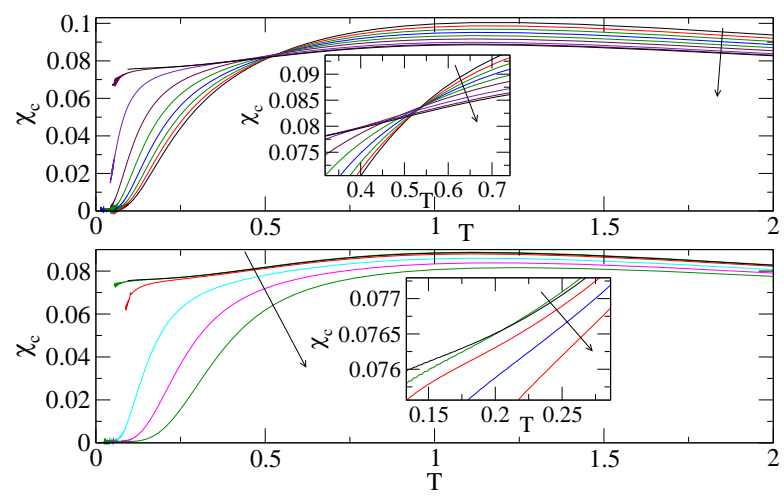

FIG. 12: (Color online) Charge susceptibility $\chi_{c}$ for $U=$ 4. Upper panel: $\chi_{c}$ for $V=1.5,1.6, \cdots, 2.1,2.15,2.17$ (in arrow direction). The inset shows a zoom of the region around the crossing point at $T^{*} \approx 0.54$. Lower panel: $\chi_{c}$ for $V=$ $2.16,2.17,2.2,2.3,2.4,2.5$ (main, in arrow direction) and $V=$ $2.16,2.17,2.18,2.19,2.2$ (inset, in arrow direction).

and $U=4$, respectively. From Fig. 11, lower panel, we see that the first curve crossing the ones for larger $V$ is the one for $V=1.04$. We therefore find $V_{c}=1.05 \pm 0.01$ for $U=2$. In principle, the critical point can be determined with this method even more accurately. Similarly for $U=4$, the inset of the lower panel of Fig. 12 shows that the first curve crossing is $V=2.16$ which leads to the estimate $V_{c}=2.165 \pm 0.005$ in this case. Both critical values are in good agreement with the most recent zero temperature DMRG calculation. ${ }^{11}$ Another interesting point is that in both cases the curves for $V<V_{c}$ do not only cross but do so at one well defined point. I.e., there is a well defined temperature $T^{*}$ where $\partial \chi_{c} / \partial V \approx 0$ for all $V$. Similar well defined crossing points have also been observed in other systems and other thermodynamic quantities, as for example, the specific heat. ${ }^{19,20}$

For the spin susceptibility, $\chi_{s}$, there is only a spin gap

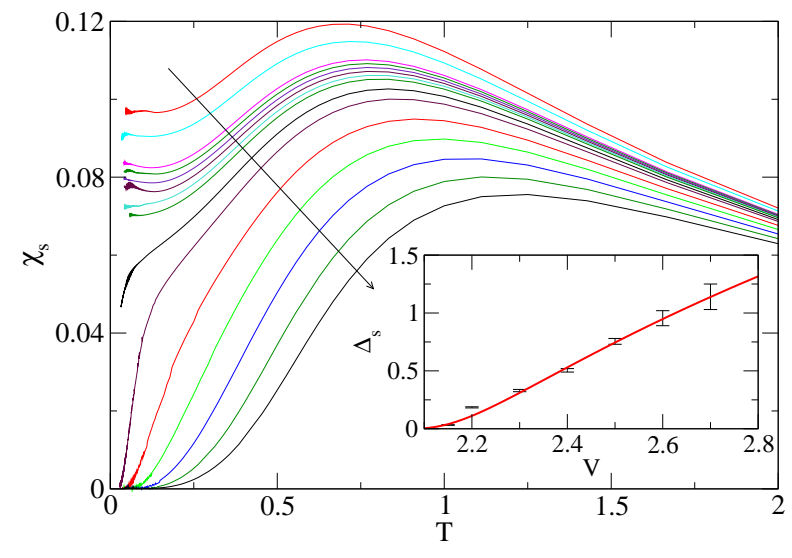

FIG. 13: (Color online) Spin susceptibility at $U=4$ for different $V=1.8,1.9,2.0,2.02, \cdots, 2.1,2.15,2.2,2.3, \cdots, 2.7$. Inset: Spin gap $\Delta_{s}$ at $U=4$ as a function of $V$. The gap is well fitted by $\Delta_{s} \sim 2.52 \sqrt{V-2.02} \cdot \exp [-0.41 /(V-2.02)]$.

above the KT transition. For $V>V_{c}^{K T}$ the temperature dependence of the spin susceptibility is then again given by Eq. (3). $\partial \chi_{s} / \partial V<0$ for all temperatures so that the curves do not cross. The same is true for $V<V_{c}^{K T}$ : In the low-temperature limit the spin sector is then described by conformal field theory and

$$
\chi_{s}(T=0)=\frac{1}{2 \pi v_{s}} .
$$

The spin velocity $v_{s}$ increases with increasing interaction strength so that again $\partial \chi_{s} / \partial V<0$ for all temperatures. Therefore no qualitative change happens at the transition line. In principle, one can try to use the fact that there is universal scaling of certain ratios of thermodynamic quantities in the conformal regime. The entropy is given by Eq. (5) so that

$$
\lim _{T \rightarrow 0} \frac{S}{T \chi_{s}} \equiv \frac{2 \pi^{2}}{3}
$$

is universal in the regime with gapless spin excitations. However, these formulas are only valid at temperatures $T \ll \Delta_{c}$. Because the spin gap opens close to the point where the charge gap vanishes, this criterion turns out to be useless for our numerical calculations. We therefore have to determine the KT line by directly extracting the gap from the susceptibility curves. As an example, we consider again the case $U=4$ (see Fig. 13). For small $V$ the behavior is qualitatively consistent with Eq. (9) whereas for large $V$ a spin gap is clearly visible. Fitting the low temperature part of the curves where a gap is present using Eq. (3) we can extract $\Delta_{s}$ as a function of $V$ as shown in the inset of Fig. 13. Here, the error bars are obtained by varying the fit region. Another fit according to Eq. (7) then yields $V_{c}^{K T} \approx 2.02 \pm 0.06$ where the error estimate stems again from a variation of the fit region. Within the estimated errors we therefore obtain strong evidence that $V_{c} \neq V_{c}^{K T}$ for $U=4$, i.e., that 


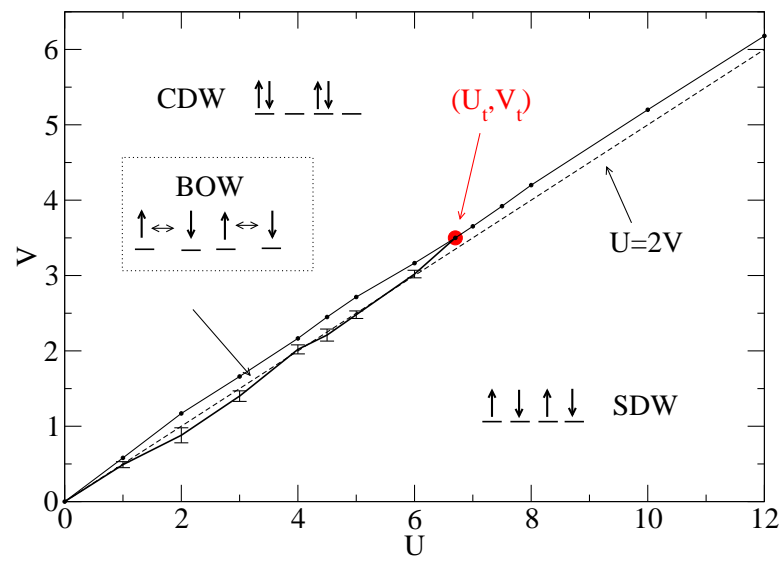

FIG. 14: (Color online) Phase diagram as obtained by TMRG. The dashed line denotes $U=2 V$. The upper line describes the phase boundary of the CDW phase. The related error is always smaller than the symbol size. The error of the KT phase transition (lower line) is shown.

we have two separate phase transitions. Following this procedure to determine the second order and the KT transition lines for other values of $U$, we obtain the phase diagram discussed in the next subsection.

\section{Phase diagram}

Our phase diagram, shown in Fig. 14, is very similar to the one obtained in the most recent zero-temperature DMRG study. ${ }^{11}$ There is a first order transition line for $(U, V)$ values above the tricritical point $\left(U_{t}, V_{t}\right)$, separating the SDW and CDW phases. Below the tricritical point we have a KT-type transition line where the spin gap opens and a second order phase transition line where the charge gap disappears. The nature of the so called BOW phase enclosed by the two transition lines is discussed in more detail in the next subsection. There is some quantitative difference between our study and Zhang's DMRG study ${ }^{11}$ in the location of the tricritical point though. We find $U_{t}=6.7 \pm 0.2, V_{t}=3.5 \pm 0.1$, whereas he found $U_{t} \approx 7.2, V_{t} \approx 3.746$. Both values are considerably larger than the ones found in QMC calculations, $U_{t}=4.7 \pm 0.1, V_{t}=2.51 \pm 0.04$ in Ref. 7, and $U_{t} \in[5,5.5]$ in Ref. 8. We also note that our phase diagram disagrees with that obtained in an earlier DMRG calculation ${ }^{10}$ where the BOW phase was restricted to the first order phase transition line (SDW-CDW) extending from above the tricritical point estimated to be at $U_{t}=3.7 \pm 0.2$ up to $U \approx 8$.

\section{E. Long-range BOW order and extent of the BOW phase}

From the phase diagram, Fig. 14, we see that the spin gap opens starting from the SDW phase and only after that the transition into the CDW phase occurs. From field theoretical considerations it is then expected that the phase enclosed by these two transition lines is a Mott state with some dimerization, also called a bond-order wave (BOW) state. Because such a dimerization does not break any continuous symmetry, true long-range order at zero temperature will occur even in one dimension. This means that for the correlation function

$$
F(r)=(-1)^{r}\left(\left\langle A_{0} A_{r}\right\rangle-\left\langle A_{r}\right\rangle^{2}\right)
$$

with $A_{r}=S_{r}^{z} S_{r+1}^{z}$ or $A_{r}=\sum_{\sigma}\left(c_{r, \sigma}^{\dagger} c_{r+1, \sigma}+\right.$ h.c. $)$ we have $\lim _{r \rightarrow \infty} F(r)=$ const $\neq 0$. With the TMRG algorithm there are different possibilities to detect this order. First, next-leading eigenvalues of the QTM allow it to calculate correlation lengths easily. In an asymptotic expansion of a two-point correlation function with operator $O_{r}$ we obtain

$$
\left\langle O_{1} O_{r}\right\rangle-\left\langle O_{1}\right\rangle\left\langle O_{r}\right\rangle=\sum_{\alpha} M_{\alpha} \mathrm{e}^{-r / \xi_{\alpha}} \mathrm{e}^{\mathrm{i} k_{\alpha} r}
$$

with correlation lengths $\xi_{\alpha}$ and wave vectors $k_{\alpha}$ given by

$$
\xi_{\alpha}^{-1}=\ln \left|\frac{\Lambda_{0}}{\Lambda_{\alpha}}\right| \quad, \quad k_{\alpha}=\arg \left(\frac{\Lambda_{0}}{\Lambda_{\alpha}}\right),
$$

where $\Lambda_{0}$ is the largest eigenvalue of the QTM and $\Lambda_{\alpha}$ another eigenvalue. A correlation length obtained according to Eq. (13) will show up in the asymptotic expansion (12) if the corresponding matrix-element $M_{\alpha}$, which can also be calculated with the TMRG algorithm, ${ }^{15,21}$ is nonzero. In the long distance limit, the behavior of the correlation function will be determined by the largest correlation length $\xi$ with nonzero matrix-element.

If the correlation function decays algebraically, this correlation length will diverge like $\xi \sim 1 / T$. If, on the other hand, the correlation function decays exponentially even at zero temperature then $\xi$ stays finite. Finally, for a correlation function showing true long-range order at zero temperature the correlation length will diverge like

$$
\xi \sim \frac{\exp (\Delta / T)}{\sqrt{T}}
$$

where $\Delta$ is the gap for the corresponding excitations.

In Fig. 15(a) we show, as an example, the leading SDW, CDW and BOW correlation lengths for $U=6$ and $V=3.16$. Here the leading SDW and CDW correlation lengths stay finite whereas the BOW correlation length diverges faster than $1 / T$ indicating long-range $\mathrm{BOW}$ order at zero temperature.

Another possibility to detect the BOW order with the TMRG algorithm is to calculate static susceptibilities

$$
\chi_{O O}(q)=\sum_{r} \mathrm{e}^{i q r} \int_{0}^{\beta} d \tau\left\langle O_{0}(0) O_{r}(\tau)\right\rangle
$$

again for some operator $O_{r}$. For true long-range order the corresponding $\chi(q)$ will diverge exponentially with temperature, whereas $\chi(q)$ will go to a constant (zero if the 


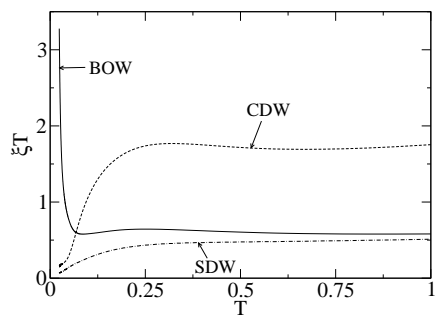

(a) $\xi T$

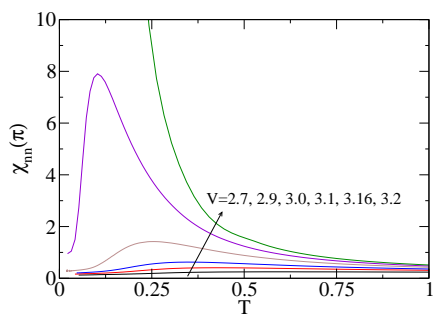

(c) $\chi_{n n}(\pi)$

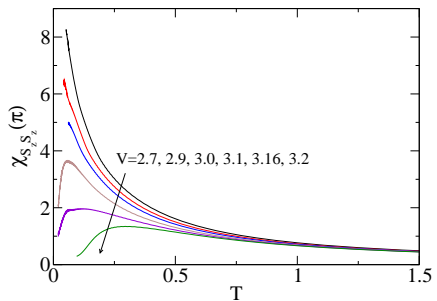

(b) $\chi_{S} z_{S} z(\pi)$

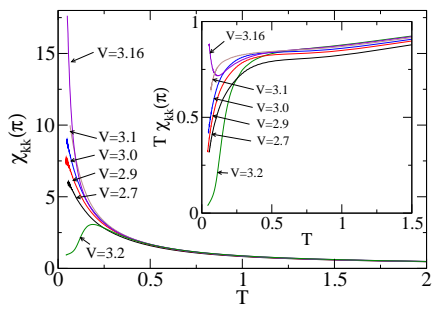

(d) $\chi_{k k}(\pi)$
FIG. 15: (Color online) (a): Leading SDW, CDW and BOW correlation lengths plotted as $\xi T$ for $U=6$ and $V=3.16$. (b)-(d): alternating static susceptibilities for the longitudinal spin, the density, and the kinetic energy $k=\sum_{\sigma}\left(c_{r, \sigma}^{\dagger} c_{r+1, \sigma}+\right.$ h.c.) for $U=6$ and different $V$, respectively.

operator is conserved) for short-range order. The situation is, however, complicated if the correlation function shows quasi long-range order, i.e., decays algebraically. Here we want to consider the case that only one sort of excitations is gapless, say the spin excitations. From conformal field theory it is known that the corresponding algebraically decaying correlation function in the longdistance limit $r \gg 1$ will behave as

$$
\begin{aligned}
\left\langle O_{0}(0) O_{r}(\tau)\right\rangle & \sim\left(\frac{2 \pi T}{v}\right)^{2 x} \exp \left[\frac{-2 \pi T x}{v} r\right] \\
& \times \exp [-\mathrm{i} k r] \exp \left[2 \pi T \mathrm{i}\left(d^{+}-d^{-}\right) \tau\right] .
\end{aligned}
$$

Here $v$ is the velocity of the elementary excitations, $x=$ $d^{+}+d^{-}$the scaling dimension, $d^{ \pm}$the conformal weights, and $k$ the characteristic wave vector. The $\tau$-integral for the static susceptibility $\chi_{O O}(k)$ can then be calculated explicitly and is given by

$$
\int_{0}^{\beta} d \tau \exp \left[2 \pi T \mathrm{i}\left(d^{+}-d^{-}\right) \tau\right]=\frac{\mathrm{e}^{2 \pi \mathrm{i}\left(d^{+}-d^{-}\right)}-1}{2 \pi \mathrm{i} T\left(d^{+}-d^{-}\right)} .
$$

If the conformal spin $s=d^{+}-d^{-}$is a non-zero integer this is the case for any type of particle-hole excitation the integral is zero and this part of the correlation function does not contribute. If, on the other hand, $s=0$ then there is no time dependence in (16) and the integral (17) yields just $1 / T$. The static susceptibility in the case of zero conformal spin will therefore scale as $\chi_{O O}(k) \sim T^{2 x-2}$. In particular, for the alternating part of the longitudinal spin-spin correlation function we have

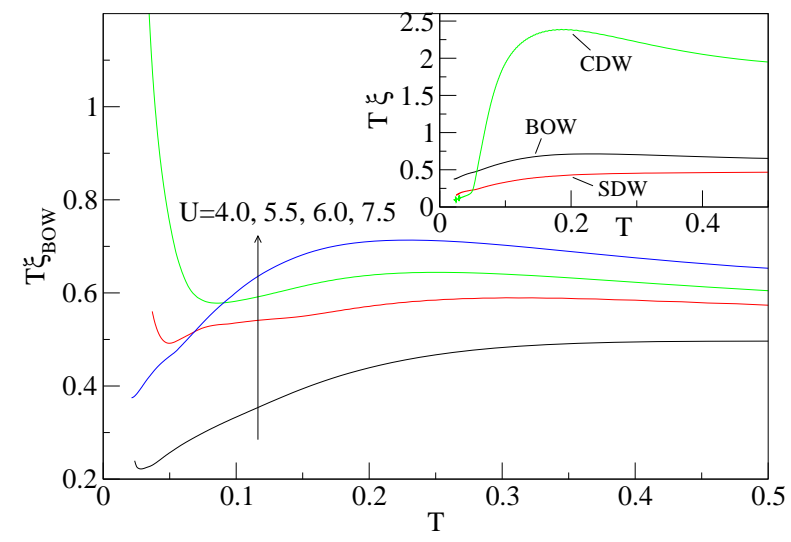

FIG. 16: (Color online) Leading BOW correlation lengths for $(U, V)=(4.0,2.14),(5.5,2.9),(6.0,3.19),(7.5,3.9)$. The inset shows the leading BOW, SDW, and CDW correlation lengths for $(U, V)=(7.5,3.9)$.

$d^{+}=d^{-}=1 / 4$ leading to $\chi_{S^{z} S^{z}}(\pi) \sim 1 / T$. Note, however, that for $x>1$ the long-distance asymptotics is no longer sufficient to discuss the behavior for $T \rightarrow 0$. In this case $\chi_{O O}(k) \rightarrow$ const for a non-conserved operator in general as in the case of exponentially decaying correlation functions discussed above.

In Figs. 15(b), 15(c), 15(d) we show for $U=6$ and different $V$ the alternating static susceptibilities for the longitudinal spin, the density, and the kinetic energy, respectively. From Fig. 15(b) we conclude that a spin gap develops for $V \gtrsim 3.1$. However, for $V=3.1$ and $V=3.16$ there is still no long-range charge order (see Fig. 15(c)), i.e., an intermediate phase does exist. In Fig. 15(d) we see that at least for $V=3.16$ this phase has longrange BOW order which is consistent with the correlation lengths shown in Fig. 15(a). Fitting the BOW correlation length using Eq. (14) we extract a rather small dimer gap $\Delta \sim 0.08$. For fixed $U$ the dimer gap is expected to decrease with decreasing $V$ so that possible long-range bond order is detected most easily close to the transition into the CDW phase. In Fig. 16 the leading BOW correlation lengths for several $U, V$-values just below this transition line are shown. For $(U, V)=(4.0,2.14),(5.5,2.9)$, and $(6.0,3.19)$ the correlation lengths diverge exponentially and we obtain the dimer gaps $\Delta \approx 0.01,0.03$, and 0.08 , respectively. As expected, $\Delta$ decreases with decreasing $U$ making it difficult to show the exponential divergence of the BOW correlation length for $U<4$ because temperatures below $T \sim 10^{-2}$ are not easily accessible by the TMRG method. Nevertheless, it is clear that the whole phase enclosed by the two transition lines shown in Fig. 14 must have long-range BOW order. For $(U, V)=(7.5,3.9)$, on the other hand, we would expect $\Delta \gtrsim 0.1$ if BOW order does exist as found in Ref. 8 so that an exponential divergence should already become obvious at $T \sim 0.1$. However, down to $T \approx 0.02$ we see no indication of such a behavior, instead the BOW correlation length seems to diverge exactly as $1 / T$ indicating 
that we are in the SDW phase. This is supported by the data in the inset of Fig. 16 showing that the leading SDW correlation length also diverges like $1 / T$ whereas the CDW correlation length stays finite for $T \rightarrow 0$. Interestingly, the BOW correlation length is larger than the SDW correlation length. We also confirmed that for $(U, V)=(7.5,3.92)$ we are already in the CDW phase. We therefore conclude that for $U=7.5$ no BOW phase exists. Instead, a direct first order transition from the SDW to the CDW phase occurs.

\section{SUMMARY AND CONCLUSIONS}

We studied the thermodynamics of the half-filled onedimensional extended Hubbard model using a TMRG algorithm. The focus was put on identifying the various phase transitions by considering thermodynamic quantities which are usually easy to measure like the uniform magnetic susceptibility, $\chi_{s}$, or the isothermal compressibility, $\chi_{c}$. For strong coupling we calculated the charge gap in the SDW as well as charge and spin gap in the CDW phase in lowest order perturbation theory. The theoretical results were confirmed by TMRG calculations of $\chi_{s}$ and $\chi_{c}$. In the weak coupling regime where the phase transitions are continuous we showed that $\chi_{c^{-}}$ curves for a fixed $U$ and different $V$ as a function of temperature cross in one well defined point if measured in the SDW or BOW phase. In the CDW phase, on the other hand, no crossing occurs. We used this criterion to determine the boundary of the CDW phase with high accuracy. The KT transition line, on the other hand, where the spin gap starts to open exponentially slowly is very difficult to determine from thermodynamic data.
Universal scaling relations obtained from conformal field theory for the magnetic susceptibility and the specific heat in the SDW phase turned out to be useless for this purpose. These scaling relations are only valid at temperatures $T \ll \Delta_{c}$ which are not accessible by TMRG because the charge gap $\Delta_{c}$ is already very small near the KT transition. We could, however, show that extracting the spin gap from the magnetic susceptibility where it is large enough and fitting it to a field theory formula does allow to determine the transition line reasonably well. In particular, the results clearly confirm that the two transition lines do not coincide and that an intermediate phase exists. By studying correlation lengths and static susceptibilities we confirmed that this additional phase has long-range bond order. We were also able to determine the tricritical point accurately and found $U_{t}=6.7 \pm 0.2, V_{t}=3.5 \pm 0.1$. Furthermore, we showed that above this point long-range bond order does not exist. Instead we find that BOW correlations can be dominant in this regime while still decaying algebraically at zero temperature. The resulting phase diagram is in good quantitative agreement with the most recent zero temperature DMRG study. ${ }^{11}$ However, it does not agree with the phase diagram found in Ref. 8 with the BOW phase existing even above the tricritical point.

\section{Acknowledgments}

The authors acknowledge helpful discussions with E. Jeckelmann and S. Nishimoto. This work has been supported by the DFG Schwerpunkt SP1073 and Graduiertenkolleg GK1052 (S.G., A.K.).
1 J. E. Hirsch, Phys. Rev. Lett. 53, 2327 (1984).

2 R. A. Bari, Phys. Rev. B 3, 2662 (1971).

3 P. G. J. van Dongen, Phys. Rev. B 49, 7904 (1994).

4 M. Nakamura, Phys. Rev. B 61, 16377 (2000).

5 J. Sólyom, Adv. Phys. 28, 201 (1979).

6 M. Nakamura, J. Phys. Soc. Jpn. 68, 3123 (1999).

7 P. Sengupta, A. W. Sandvik, and D. K. Campell, Phys, Rev. B 65, 155113 (2002).

8 A. W. Sandvik, L. Balents, and D. K. Campbell, Phys. Rev. Lett. 92, 236401 (2004).

9 M. Tsuchiizu and A. Furusaki, Phys. Rev. Lett. 88, 056402 (2002).

10 E. Jeckelmann, Phys. Rev. Lett. 89, 236401 (2002).

11 Y. Z. Zhang, Phys. Rev. Lett. 92, 246404 (2004).

12 K.-M. Tam, S.-W. Tsai, and D. K. Campbell, Phys. Rev. Lett. 96, 036408 (2006).

13 I.Peschel, X. Wang, M. Kaulke, and K. Hallberg, eds., Density-Matrix Renormalization, Lecture Notes in Physics, vol. 528 (Springer, Berlin, 1999), and references therein.

14 S. Glocke, A. Klümper, and J. Sirker, cond-mat/0610689 (2006).

15 J. Sirker and A. Klümper, Europhys. Lett. 60, 262 (2002).

16 G. Jüttner, A. Klümper, and J. Suzuki, Nucl. Phys. B 522, 471 (1998).

17 G. Jüttner, A. Klümper, and J. Suzuki, Nucl. Phys. B 487, 650 (1997).

18 M. Nakamura, A. Kitazawa, and K. Nomura, Phys. Rev. B 60, 7850 (1999).

19 D. Vollhardt, Phys. Rev. Lett. 78, 1307 (1997).

20 A. Kemper and A. Schadschneider, Phys. Rev. B 68, 235102 (2003).

21 J. Sirker and A. Klümper, Phys. Rev. B 66, 245102 (2002). 